\title{
CO deficiency in galaxies of the Fornax cluster?
}

\author{
Cathy Horellou1, Fabienne Casoli2,1, Christophe Dupraz ${ }^{1,2}$
}

\section{N93-26\%91}

1 DEMIRM, Observatoire de Paris, Section de Meudon, F-92195 Meudon Cedex, France

2 Ecole Normale Supérieure, 24 rue Lhomond, F-75231 Paris Cedex 05, France

There is ample observational evidence that cluster galaxies are different from those in the field. Interaction with the hot intracluster medium affects the morphology of the galaxies, their gaseous content and possibly their star-formation activity. Tidal encounters between galaxies also play an important role.

The atomic component has been investigated in detail for several clusters, among them our neighbour Virgo. A large fraction of the bright Virgo spirals have about one order of magnitude less HI than isolated counterparts of same optical size and type (Giovanelli \& Haynes : 1983, AJ $88,881)$. What about the molecular phase of the interstellar medium in cluster galaxies? Molecular clouds are the raw material for star formation. CO surveys of Virgo bright galaxies (Stark et al. : 1986, ApJ 310, 660 ; Kenney \& Young : 1989, ApJ 344, 171) do not show any evidence of $\mathrm{H}_{2}$ deficiency : the mechanisms tearing out the low-density atomic gas leave intact the denser molecular clouds. A similar result has recently been found for the much denser and more distant Coma cluster (Casoli et al. : 1991, A\&A 249, 359). To date, CO observations are available for those two rich clusters only.

With the Swedish-ESO $15 \mathrm{~m}$ telescope, we have observed in the ${ }^{12} \mathrm{CO}(1-0)$ transition the 23 brightest spirals and lenticulars of the Fornax cluster. Fornax is the nearest southern cluster of galaxies, at the same distance as Virgo $\left(D=17 \mathrm{Mpc}\right.$ adopted, consistent with $\mathrm{H}_{\mathrm{o}}=75 \mathrm{~km} \mathrm{~s}^{-1}$ $\mathrm{Mpc}^{-1}$ ). Fornax contains 7 times less galaxies than Virgo, but its central regions are 2.5 times denser. The spatial resolution of the $\mathrm{CO}$ observations is $44^{\prime \prime}$, corresponding to a linear distance of $3.6 \mathrm{kpc}$ at $17 \mathrm{Mpc}$. Every galaxy with an optical diametre larger than $3^{\prime}$ was mapped at half beamwidth along the major axis, even if no $\mathrm{CO}$ was detected towards the centre. Since every extended galaxy of the sample happens to have a high inclination, we expect not to miss any $\mathrm{CO}$ emission. With the Nançay radiotelescope, we also measured the $\mathrm{HI}$ emission of 11 galaxies of our sample. The resolution of the HI observations is $4^{\prime}(\mathrm{E}-\mathrm{W})$ by $21^{\prime}(\mathrm{N}-\mathrm{S})$.

Only 9 galaxies out of 23 were detected in $\mathrm{CO}$, despite long integration times (typically 80 minutes) and the low noise level (we could detect $\mathrm{H}_{2}$ masses as low as $510^{7} \mathrm{M}_{\mathrm{o}}$ ). The average CO emission of Fornax galaxies is 8 times lower than in a template sample based on the FCRAO extragalactic survey. Figure 1 displays the $\mathrm{M}\left(\mathrm{H}_{2}\right) / \mathrm{D}^{2}$ ratio for the Fornax (triangles) and template (circles) galaxies versus their morphological type. 


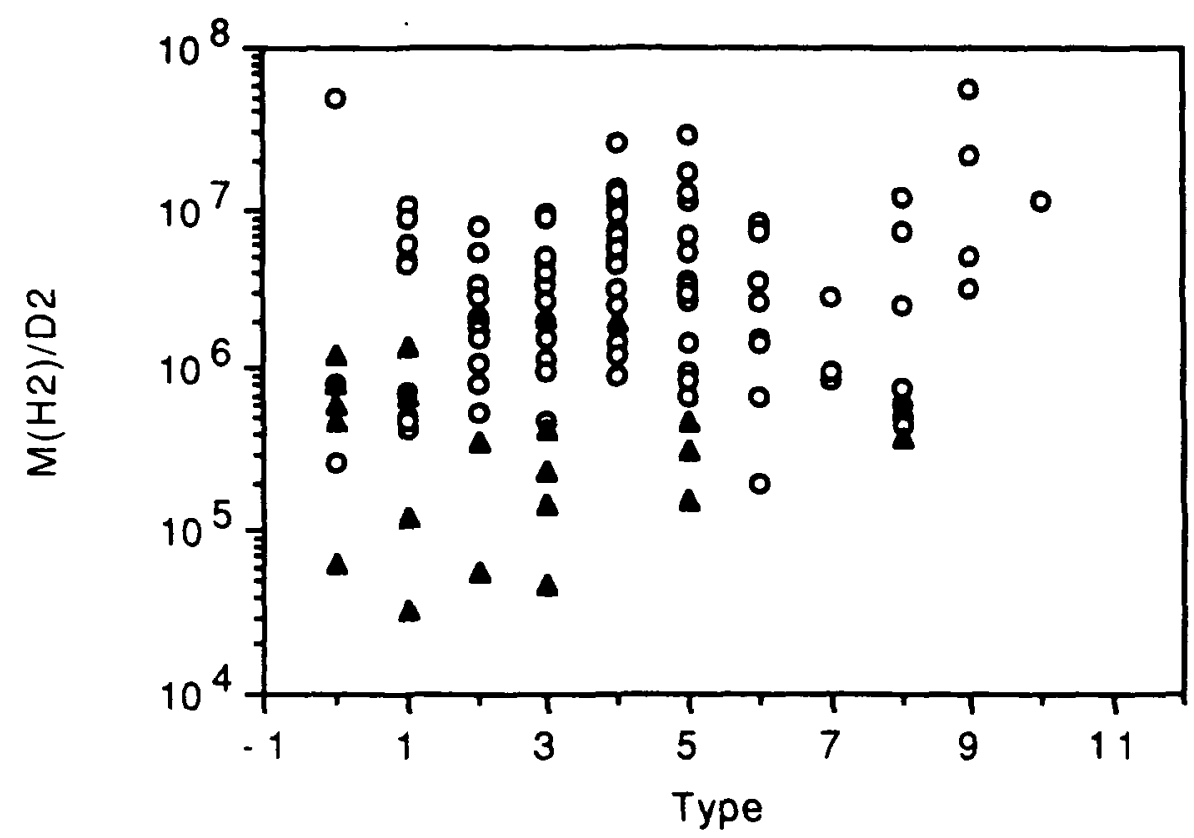

Figure 1 : Plot of the $\mathrm{M}\left(\mathrm{H}_{2}\right) / \mathrm{D}^{2}$ ratio versus galaxy morphological type, for the Fornax sample galaxies (triangles) and the template FCRAO objects (circles).

In HI, we have detected 10 galaxies out of 11 , and the HI masses of the other 12 objects were taken from the literature. Surprisingly, the HI emission of the Fornax galaxies is normal: this result is hard to explain, because all of the mechanisms which can be invoked (ram pressure stripping, evaporation, collisions between galaxies) affect the diffuse and extended atomic gas much more efficiently than the denser molecular gas.

Are the Fornax galaxies genuinely deficient in molecular gas, or must we suspect the conversion factor from $\mathrm{CO}$ emissivities to $\mathrm{H}_{2}$ column densities? As for the latter, we used the value $\mathrm{N}\left(\mathrm{H}_{2}\right) / \mathrm{I}(\mathrm{CO})=2.310^{20} \mathrm{~cm}^{-2}\left(\mathrm{~K} \mathrm{~km} \mathrm{~s}^{-1}\right)^{-1}$ derived for the Milky Way by Strong et al. (1988, A\&A 207, 1). The molecular masses may be underestimated if the Fornax galaxies have a low metallicity, but certainly not by a factor as large as 8 . Indeed, even in the metal-poor Magellanic Clouds, the correction factor does not exceed 2 (Johansson : 1991, IAU 146, 1). Furthermore, our Fornax sample galaxies are more luminous than the LMC, thus more massive and metallic, and other indicators such as the color indices $\mathrm{U}-\mathrm{B}$ and $\mathrm{B}-\mathrm{V}$ or the FIR luminosity are consistent with a low star-formation activity.

The low $\mathrm{CO}$ emission can be partly due to the small size of the Fornax galaxies with respect to the template sample, but this effect alone cannot account for the observed deficiency.

In conclusion, we rather suggest that the reference sample, as selected from the infrared, is strongly biased towards $\mathrm{CO}$-rich galaxies. Could the Fornax galaxies be more representative of "normal" galaxies? Be what it may, our result stresses the need for CO observations of optically-selected isolated galaxies, in order to determine the molecular content of "normal" galaxies. 